\title{
Prevalence and profile of sleep disordered breathing amongst patients with congestive heart failure
}

\author{
J. C. Suri*, Manish Sharma**, Geeta Kampani** , M. K. Sen* \\ * Department of Pulmonary, Critical Care \& Sleep Medicine, ** Department of Medicine, \\ Vardhman Mahavir Medical College \& Safdarjang Hospital, New Delhi \\ Indian J Sleep Med 2010; 5.4, 120-127
}

\begin{abstract}
Introduction: It has been observed that since heart failure is highly prevalent and central sleep apnea (CSA) is common in patients with a failing heart, heart failure is the commonest cause of CSA in the general population.

Aims \& Objectives: The present study was undertaken with the purpose of finding prevalence of sleep disordered breathing (SDB) in patients of heart failure and also to find the association of severity of SDB with severity of heart failure.

Material \& Methods: Forty patients suffering from systolic heart failure were selected on random basis. All these patients underwent complete evaluation of history, physical examination and overnight polysomnography. The patients were divided into two groups, namely group 1 and group 2, on the basis of polysomnography. Group 1 consisted of 17 patients who did not have sleep disordered breathing i.e. AHI (central or obstructive) < 5. Group 2 consisted of 23 patients who had sleep disordered breathing i.e. AHI (central or obstructive) $>5$. Comparison of biochemical profile and sleep parameters was made between group 1 and group 2 and results analyzed.

Observations: Aetiology of heart failure was ischemic heart disease in 34 patients, viral myocarditis in 3 patients and postpartum cardiomyopathy in 3 patients. Total prevalence of CSA in heart failure was $57.5 \%$.Prevelance in males and females was $47.6 \%$ and $68.42 \%$ respectively. There was a significant difference in $\mathrm{O} 2$ desaturation index, minimum $\mathrm{O} 2$, arousal index, total sleep time, $\mathrm{AHI}$ (central), sleep efficiency and wake $\mathrm{O} 2$ amongst the two groups. A negative correlation was observed between ejection fraction and $\mathrm{O} 2$ desaturation index, $\mathrm{AHI}$ (central), and arousal index. A positive correlation was found between ejection fraction and wake $\mathrm{O} 2$.

Conclusions: A fairly high prevalence of sleep-disordered breathing (57.5\%) was found in patients of heart failure in the present study. With increasing severity of HF a significant worsening of CSACSR was observed. The treatment of CSA-CSR may prevent the worsening status of HF. Hence long term randomized and controlled interventions are required to further substantiate these fact.
\end{abstract}

Keywords: sleep disordered breathing, central sleep apnea, Cheyne-Stokes respiration, congestive heart failure

Address for correspondence

Prof (Dr) J. C. Suri

Consultant \& Head, Dept of Pulmonary,

Critical Care \& Sleep Medicine, Vardhman

Mahavir Medical College \& Safdarjang Hospital,

New Delhi, India. Email: jcsuri@rediffmail.com

Indian Journal of Sleep Medicine (IJSM), Vol. 5, No. 4, 2010 


\section{Introduction}

$\mathrm{C}$ entral sleep apnea (CSA) is characterized by a loss of ventilator effort lasting for 10 seconds or pontomedullary pacemaker that generates the breathing rhythm.(24) Poly- somnographically, there is cessation of airflow at mouth as well as absence of thoracoabdominal excursions. There are several physiological and pathological conditions associated with the occurrence of CSA. Physiologic conditions encountered are sleep onset, post-arousal/ post-sigh phase and phasic REM sleep. The various pathologic states include nonhypercapneic CSA (systolic heart failure, post-stroke and high altitude), hypercapneic CSA (primary and congenital hypoventilation syndromes, brainstem and spinal cord disorders like encephalitis, tumors, infarcts, amyotrophic lateral sclerosis, muscular disorders and Guillain-Barre syndrome), endocrinopathies (like acromegaly and hypothyroidism) and upper airway disorders. CSA has also been described to occur in cases of obstructive sleep apnea (OSA), particularly when the latter is treated with CPAP, when it is termed as complex sleep apnea. By and large, it has been observed that since heart failure is highly prevalent and CSA is common in patients with a failing heart, heart failure is the commonest cause of CSA in the general population. (24)

Congestive heart failure $(\mathrm{CHF})$ is a clinical syndrome that occurs in patients who, because of an inherited or acquired abnormality of cardiac structure and /or function, develop a constellation of clinical symptoms (dyspnoea and fatigue) and signs (oedema and rales) that lead to frequent hospitalizations, poor quality of life, and shortened life expectancy.(1) Conventionally, heart failure has been known to be associated with sleep disordered breathing (SDB)(19).CHF may occur due to valvular disease, cardiac arrhythmias or cardiac muscle pump failure. Cardiac muscle pump failure or cardiomyopathy is due to systolic or diastolic dysfunction. The former occurs as a result of impaired left ventricular contractility (left ventricular ejection fraction $<40 \%$ or impaired left ventricular shortening $<28 \%$ ), usually secondary to ischemic heart disease, longstanding hypertension or unknown causes (idiopathic). Diastolic dysfunction, also known as heart failure with normal systolic function (HFNSF), occurs due to stiff ventricular walls due to hypoxia, tachycardia, hypertension, and rarely following constrictive pericarditis, pericardial effusion and myocardial infiltrative disorders. (2,3) HFNSF is responsible for CHF in 13 to $74 \%$ of patients.(4) According to a recent report, in a combined group of 1250 consecutive patients with systolic heart failure, using apnea-hypopnea index (AHI) of 15 as the threshold, $52 \%$ of the patients were found to have moderate to severe sleep apnea, 31\% had central sleep apnea, and $21 \%$ had obstructive sleep apnea.(24)

Sleep disordered breathing represents a spectrum of abnormalities that range from mild (simple snoring) to severe (hypoventilation and sleep apnoea-hypopnoea) forms. SDB can manifest as obstructive sleep apnoea (OSA) and central sleep apnoea (CSA). In patients with heart failure, Cheyne - Stokes respiration (CSR), a periodic form of crescendodecrescendo breathing, is synonymous with CSA $(5,18)$.

\section{Aims \& objectives}

The present study was undertaken in the Department of Pulmonary, Critical Care and Sleep Medicine, Safdarjung Hospital, New Delhi with the purpose of finding prevalence of sleep disordered breathing (SDB) in patients of heart failure and also to find the association of severity of SDB with severity of heart failure. Not much is known about the prevalence of sleep disordered breathing in patients of heart failure in India.

\section{Material \& Methods}

In this study 40 patients suffering from systolic heart failure were selected on random basis. Patient enrolment was independent of the presence of sleep-related symptoms (i.e. snoring, witnessed apnoeas, excessive daytime sleepiness) although detailed history of all these symptoms was sought. All these patients underwent complete evaluation of history, physical examination and overnight polysomnography. The patients were divided into two groups, namely group 1 and group 2, on the basis of polysomnography. Group 1 consisted of 17 patients who did not have sleep disordered breathing i.e. AHI (central or obstructive) < 5. Group 2 consisted of 23 patients who had sleep disordered breathing i.e. AHI (central or obstructive) $\geq 5$. Comparison of biochemical profile and sleep parameters was made between group 1 and group 2 and results analyzed. As all the patients were dyspneic (either NYHA class 2 or NYHA class 3 ), these two groups of patients were compared for their sleep parameters. Further sub-grouping of patients was done into four subgroups according to number of drugs (beta blocker, ACE inhibitor, nitrates, diuretics or digoxin) being taken by the patient. Group A consisting of patients taking one drug, group $\mathrm{B}$ consisting of patients on two drugs, group $\mathrm{C}$ 
consisting of patients on three drugs and group D consisting of patient on four drugs. No patient was on all five drugs. An attempt was made to establish the correlation between the number of drugs being taken by the patient and the various sleep parameters.

\section{Diagnosis of OSA and CSA}

The diagnosis was made in accordance with the guidelines of AASM Manual for scoring sleep, 2007 as following.(4) Accordingly the various events were defined as follows.

Apnea: Apnea was scored when all of the following criteria were met (a) drop in peak thermal sensor excursion by >=90\% of the baseline, (b) duration of event lasts for at least 10 seconds, and (c) at least $90 \%$ of the event's duration meets the amplitude reduction criteria for apnea.

Obstuctive apnea: Apnea was classified as obstructive if it was associated with increased or continued effort throughout the entire period of absent airflow.

Central apnea: Apnea was classified as central if it was associated with absent inspiratory effort throughout the entire period of absent airflow. The patient reports at least one of the following (a) excessive daytime sleepiness, (b) frequent arousals and awakenings during sleep or insomnia complaints, and (c) awakening with shortness of breath. Polysomnography shows five or more central apneas per hour of sleep. The disorder is not better explained by another current sleep disorder, medical or neurological disorder, medication use, or substance use disorder. (23)

Cheyne Stokes breathing pattern: Polysomnography shows at least 10 central apneas and hypopneas per hour of sleep in which the hypopnea has a crescendo-decrescendo pattern of tidal volume accompanied by frequent arousals from sleep and derangement of sleep structure. Although symptoms are not mandatory to make this diagnosis, patients often report excessive daytime sleepiness, frequent arousals and awakenings during sleep, insomnia complaints, or awakening with shortness of breath. The breathing disorder occurs in association with a serious medical illness, such as heart failure, stroke, or renal failure. The disorder is not better explained by another current sleep disorder, medical or neurological disorder, medication use, or substance use disorder. (23)

Mixed apnea: Apnea was scored mixed apnea if it met apnea criteria and was associated with absent inspiratory effort in the initial portion of the event, followed by resumption of inspiratory effort in the second portion of the event.

Hypopnea: hypopnea was scored if ALL of the following criteria were met: (a) the nasal pressure signal excursions (or alternative hypopnea sensor) drop by $>30 \%$ of baseline, (b) the duration lasted at least 10 seconds, (c) less than $4 \%$ desaturation from pre-event baseline, and (d) at least 90\% of the event's duration must meet the amplitude reduction of criteria for hypopnea.

Respiratory effort related arousal: Respiratory effort-related arousal (RERA) was scored if there was a sequence of breaths lasting at least 10 seconds characterized by increasing respiratory effort or flattening of the nasal pressure waveform leading to an arousal from sleep when the sequence of breaths does not meet criteria for an apnea or hypopnea.

Arousals: Arousal was scored during any stage of sleep if there was an abrupt shift of EEG frequency including alpha, theta, and/or frequencies $>16 \mathrm{~Hz}$ (but not spindles) that lasts at least 3 seconds, with at least 10 seconds of stable sleep preceding the change. The number of arousals per hour is taken as the arousal index (AI).

Desaturations: Desaturation is defined as a fall $>3 \%$ in saturation from baseline following a respiratory event. The number of such episodes per hour is taken as desaturation index.(DI).

Apnea Hypopnea Index (AHI): The AHI was defined as the number of apneic and hypopneic episodes per hour of sleep.

Diagnosis of OSA was based on polysomnography with an AHI (obstructive) of $>5$ per hour. Diagnosis of CSA was based on polysomnography with an AHI (central) of $>5$ per hour.

Diagnosis of systolic heart failure was made by $2 \mathrm{D}$ echocardiography. Patients with left ventricular ejection fraction $<40 \%$ were included in study. Patients with congenital heart diseases, unstable angina, acute pulmonary edema, intrinsic liver and renal diseases, kyphoscoliosis, intrinsic pulmonary diseases, patients on theophylline and morphine and its derivatives were excluded from the study. All subjects underwent detailed history taking, clinical examination, examination of previous medical records and relevant laboratory investigations.

A whole night fully supervised, manually validated level1 polysomnography was conducted. It included electroencephalography, electro-oculography, chin electromyography, oro-nasal airflow (by nasal thermistors and nasal pressure transducer ), rib cage and abdominal movements, arterial oxygen saturation monitored via a finger probe, electrocardiography and body position. An 
Alice 5 Healthdyne polysomnography system (Respironics, USA) was used. Sleep staging was performed using the criteria of AASM manual for scoring sleep 2007 as described above. A trained physician manually validated all the sleep studies. The minimum arterial blood oxygen saturation attained during sleep was also recorded. Categorization of severity of CSA is done according to AHI (central) value into three groups; mild (5-15), moderate (16-30) and severe (>30).

\section{Statistical Methods}

The observed data for the various biochemical parameters was presented in terms of mean \pm standard deviation (S.D.) for a descriptive analysis. Further analysis of the data for categorical variables was done using the Chi-square test. Logistic regression was applied for BMI adjustment. The level of statistical significance was taken as P d" 0.05. The data was analyzed by using the SPSS statistical software, version 15.0.

\section{Observations \& Results}

Forty patients of heart failure with ejection fraction $<40 \%$ were studied. Out of 40 patients 15 patients were on nitrates, 14 patients were on digoxin, 26 patients were on ace inhibitors, 23 on diuretics and 30 patients were on beta blockers. Aetiology of heart failure was ischemic heart disease in 34 patients, viral myocarditis in 3 patients and postpartum cardiomyopathy in 3 patients (Table 3). Total prevalence of CSA in heart failure was $57.5 \%$.Prevelance in males and females was $47.6 \%$ and $68.42 \%$ respectively. (Table 1)

The prevalence of OSA was found to be $12.5 \%$ (Table 2)

There was significant difference ( $\mathrm{p}$ Value $=.006)$ in ejection fraction in group $1(29.96 \pm 4.9 \%)$ and group 2 $(35.82 \pm 3.5)$. In addition there was also a significant difference in SBP ( $p$ Value $=0.042$ ). SBP being $117.53 \pm$ 9.23 in group 1 compared to $124.52 \pm 11.7$ in Group 2 . (Table 4)

There was a significant difference in symptoms, O2 desaturation index, minimum $\mathrm{O} 2$, arousal index, total sleep time, AHI (central), sleep efficiency and wake $\mathrm{O} 2$ as indicated by $\mathrm{p}$ values (Tables $5 \& 6$ )

A negative correlation was observed between ejection fraction and $\mathrm{O} 2$ desaturation index, AHI (central), and arousal index. A positive correlation was found between
Table 1: Total prevalence of central sleep apnea in the cases

\begin{tabular}{|l|c|c|c|c|}
\hline & $\begin{array}{c}\text { HF with } \\
\text { CSA }\end{array}$ & $\begin{array}{c}\text { HF } \\
\text { without } \\
\text { CSA }\end{array}$ & $\begin{array}{c}\text { Total } \\
\text { Patients } \\
\text { of HF }\end{array}$ & $\begin{array}{c}\text { Percentage } \\
\text { of HF with } \\
\text { CSA }\end{array}$ \\
\hline Male & 10 & 11 & 21 & $47.6 \%$ \\
\hline Female & 13 & 6 & 19 & $68.42 \%$ \\
\hline Total & 23 & 17 & 40 & $57.5 \%$ \\
\hline
\end{tabular}

(HF = Heart failure; CSA = Central Sleep apnea)

Table 2: Prevalence of obstructive sleep apnea in the cases

\begin{tabular}{|c|c|c|c|}
\hline $\begin{array}{c}\text { Total } \\
\text { HF Cases }\end{array}$ & $\begin{array}{c}\text { HF without } \\
\text { OSA }\end{array}$ & $\begin{array}{c}\text { HF } \\
\text { with OSA }\end{array}$ & Percentage \\
\hline 40 & 35 & 5 & $12.5 \%$ \\
\hline
\end{tabular}

Table 3: Etiology of heart failure among patients

\begin{tabular}{|l|c|}
\hline Etiology & Number of Patients \\
\hline Ischemic Cardiomyopathy & 34 \\
\hline Post Partum Cardiomyopathy & 3 \\
\hline Viral Myocarditis & 3 \\
\hline
\end{tabular}

Table 4: Comparison of demographic characteristics between patients without CSA (Group 1) and patients with CSA (Group 2)

\begin{tabular}{|c|c|c|c|}
\hline Characteristics & Group 1 & Group 2 & Pvalue \\
\hline Age (years) & $44.76 \pm 13.46$ & $52.39 \pm 9.8$ & 0.058 \\
\hline Height $(\mathrm{cm})$ & $163.59 \pm 7.45$ & $159.78 \pm 8.22$ & 0.135 \\
\hline Weight (Kgs) & $62.65 \pm 7.5$ & $62 \pm 10.2$ & 0.819 \\
\hline BMI & $23.1 \pm 1.59$ & $24.2 \pm 3.0$ & 0.165 \\
\hline $\begin{array}{l}\text { Duration of heart } \\
\text { failure (months) }\end{array}$ & $18.53 \pm 15.4$ & $15.7 \pm 13.6$ & 0.552 \\
\hline Ejection fraction $\%$ & $35.82 \pm 3.5$ & $29.96 \pm 4.9$ & 0.006 \\
\hline SBP $(\mathrm{mm}$ of $\mathrm{Hg})$ & $117.53 \pm 9.23$ & $124.52 \pm 11.7$ & 0.042 \\
\hline $\mathrm{DBP}(\mathrm{mm}$ of $\mathrm{Hg})$ & $76.35 \pm 5.6$ & $81.13 \pm 9.3$ & 0.053 \\
\hline Hemoglobin Gram\% & $12.6 \pm 1.73$ & $13.56 \pm 1.2$ & 0.06 \\
\hline $\begin{array}{l}\text { Platelets (in Lakhs } \\
\text { per cu mm) }\end{array}$ & $2.54 \pm 0.67$ & $2.46 \pm 0.57$ & 0.67 \\
\hline TLC (per cu mm) & $\begin{array}{l}6652.94 \pm \\
1597.6\end{array}$ & $\begin{array}{l}6434.78 \pm \\
1273.3\end{array}$ & 0.646 \\
\hline $\begin{array}{l}\text { Blood urea } \\
(\mathrm{mg} \text { per } \mathrm{dl})\end{array}$ & $25.82 \pm 10.6$ & $27.04 \pm 9.2$ & 0.701 \\
\hline $\begin{array}{l}\text { S. creatinine } \\
(\mathrm{mg} \text { per } \mathrm{dl})\end{array}$ & $1.2 \pm 2.5$ & $1.843 \pm 0.199$ & 0.701 \\
\hline $\begin{array}{l}\text { Random blood sugar } \\
\text { (mg per dl) }\end{array}$ & $140 \pm 14$ & $142 \pm 15.5$ & 0.61 \\
\hline
\end{tabular}

Indian Journal of Sleep Medicine (IJSM), Vol. 5, No. 4, 2010 
ejection fraction and wake O2. (Figure 1)

Table 5: Comparison of symptoms of SDB between Group 1 and Group 2

\begin{tabular}{|l|c|c|}
\hline \multicolumn{1}{|c|}{ Symptom } & $\begin{array}{c}\text { Percentage } \\
\text { of patients in } \\
\text { Group 1 }\end{array}$ & $\begin{array}{c}\text { Percentage of } \\
\text { patients in } \\
\text { Group 2 }\end{array}$ \\
\hline Excessive daytime sleepiness & 14 & 26 \\
\hline Witnessed apneas & 12 & 25 \\
\hline Daytime fatigue & 15 & 23 \\
\hline
\end{tabular}

Table 6: Comparison of sleep parameters between Group 1 and Group 2

\begin{tabular}{|l|l|l|l|}
\hline Characteristics & Group 1 & Group 2 & p value \\
\hline O2 desaturation index & $4.86 \pm 3.8$ & $30.62 \pm 23.4$ & $<0.01$ \\
\hline Minimum O2\% & $90.8 \pm 3.7$ & $75.3 \pm 19.4$ & 0.03 \\
\hline Arousal index & $12.41 \pm 5.6$ & $22.6 \pm 12.7$ & 0.002 \\
\hline $\begin{array}{l}\text { Time in bed } \\
\text { (in minutes) }\end{array}$ & $392 \pm 25$ & $400 \pm 19$ & 0.594 \\
\hline $\begin{array}{l}\text { Total sleep time } \\
\text { (in minutes) }\end{array}$ & $300 \pm 64$ & $277 \pm 49$ & $<0.05$ \\
\hline $\begin{array}{l}\text { Sleep efficiency (total } \\
\text { sleep time/time in bed \%) }\end{array}$ & $76.5 \pm 15$ & $69 \pm 13$ & $<0.05$ \\
\hline $\begin{array}{l}\text { AHI (central) } \\
\text { wake O2\% }\end{array}$ & $2.08 \pm 1.5$ & $28.15 \pm 20.9$ & $<0.01$ \\
$94.8 \pm 2.84$ & $90.6 \pm 3.0$ & $<0.01$ \\
\hline
\end{tabular}
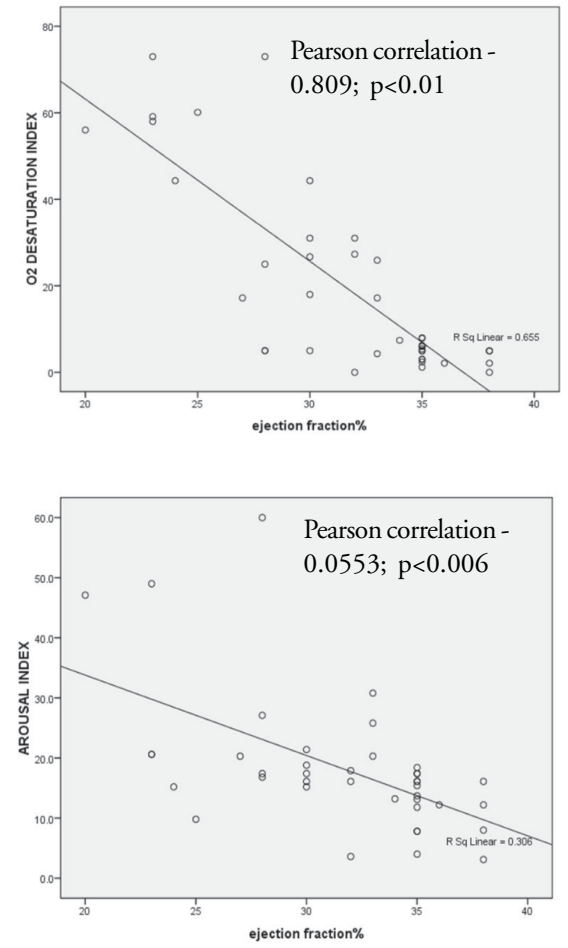

Significant difference was observed in various sleep parameters in group $\mathrm{A}, \mathrm{B}, \mathrm{C}$ and $\mathrm{D}$ as indicated by the $\mathrm{p}$ values. (Table 7)

\section{Discussion}

\section{Prevalence of SDB in heart failure}

In our study we found the prevalence of sleep disordered breathing in patients of heart failure to be $57.5 \%$ i.e. 23 out of 40 patients. All the 23 patients had CSA. There was no significant gender difference in the prevalence of SDB amongst the patients. Out of 23 patients who had CSA 5 (12.5\%) patients were also found to have OSA (Table-2). The prevalence of SDB in the study conducted by Jawaheri et al on 81 ambulatory male patients of heart failure was $51 \%$ (40\% had CSA and 11\% had OSA) (6). In another study conducted by Schulz et al, the prevalence was found to be $43 \%$ and $28 \%$ for OSA and CSA respectively in patients of heart failure (7). The prevalence of SDB in the study conducted by Sonia Ancoli - Israel was 53\% in patients of heart failure.(8) The mean AHI (central) in our present study was $28.15 \pm 20.9$ while that in study conducted by Schulz, Jawaheri and Sonia Ancoli was 35 \pm 3, $44 \pm 19$ and $30 \pm 5$ respectively $(6,7)$. The prevalence of SDB in our $\mathrm{CHF}$
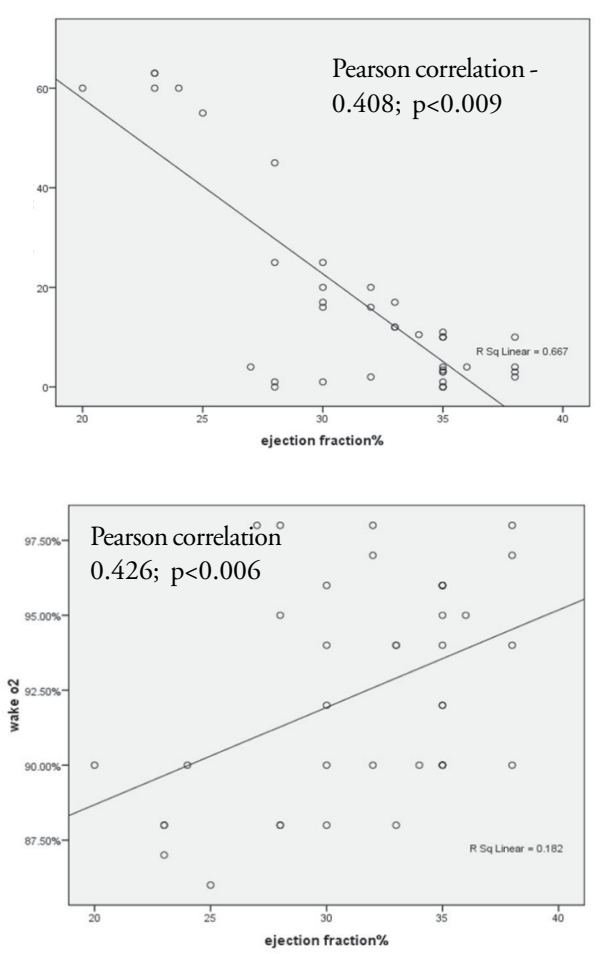

Figure 1: Correlation of ejection fraction and $\mathrm{O} 2$ desturation index, AHI (central), arousal index and wake O2. 
Table 7: Comparison of various sleep parameters in Group A, B, C and D

\begin{tabular}{|l|l|l|l|l|l|}
\hline Sleep parameter & Group A & Group B & Group C & Group D & PValue \\
\hline O2 deaturation index & $14.19 \pm 26.0$ & $8.55 \pm 8.5$ & $26.49 \pm 23.9$ & $27.68 \pm 23.4$ & 0.12 \\
\hline Arousal index & $13.8 \pm 7.3$ & $15.4 \pm 6.4$ & $19.3 \pm 10.8$ & $23.7 \pm 17.5$ & 0.28 \\
\hline Apnea hypopnea index (central) & $11.7 \pm 15.2$ & $8.49 \pm 6.9$ & $20.3 \pm 23.8$ & $27.1 \pm 26.2$ & 0.17 \\
\hline Minimum O2 & $76 \pm 16$ & $78 \pm 20.8$ & $70.5 \pm 22.7$ & $82 \pm 18.9$ & 0.34 \\
\hline Sleep quality & $87.1 \pm 6.3$ & $79.9 \pm 17.8$ & $87.2 \pm 7.5$ & $85.9 \pm 5.7$ & 0.31 \\
\hline Wake O2 & $91.7 \pm 2.8$ & $94.0 \pm 3.3$ & $92.4 \pm 4.1$ & $91.1 \pm 3.4$ & 0.34 \\
\hline
\end{tabular}

patients was higher. However, it is difficult to compare the results of the present study to those obtained in earlier studies for several reasons. Various studies have employed different scoring criteria for SDB; the sleep recordings from the different studies have been scored by different investigators, thus introducing inter-rater variability. There have been differences in monitoring techniques of sleep and breathing between various studies. For example, in contrast to the our study and that conducted by Jawaheri et al which mainly employed in-hospital polysomnography, Schulz used a portable device which may underestimate the prevalence and severity of the disease. (7) The criteria for defining the heart failure also vary in different studies, for example, we selected patients with ejection fraction less than $40 \%$ while Jawaheri et al had enrolled patients with ejection fraction less than $45 \%$. Moreover, the profile of the population studied and the number of patients were also different in different studies. Furthermore, the mean age of patients in different studies was also different. It may be appreciated that the various studies were performed in different regions of the world and ethnicity may influence the occurrence of SDB due to differences in respiratory chemosensitivity, craniofacial morphology and the level of obesity.

Similar to the study conducted by Jawaheri we also found in our study a higher prevalence of CSA as compared to OSA in contrast to the study done by Schulz which found more prevalence of OSA as compared to CSA. It is speculated that the optimization in pharmacotherapy in the form of beta blocker led to the disappearance of CSR in a subset of patients leading to decreased prevalence of CSA as compared to OSA in study conducted by Schulz. It is worth noting that, in the study conducted by Jawaheri, none of the patients was on beta blockers while percentage of patients on bêta blockers in study by Schulz was $90 \%$. The proportion of patients on beta blockers in our study was $45 \%$. Beta blockers might suppress CSR not only by decreasing circulation times and pulmonary venous congestion but also by altering chemo-reflex regulation of ventilatory control. It is worth noting that this assumption is supported by two recent studies showing that $\mathrm{CHF}$ patients taking â-blockers have a lower prevalence and severity of CSR than those who do not (9-11). No significant difference was found in sleep characteristics of the patients on different drugs although 18 out of 40 patients i.e. $45 \%$ of our patients were on beta blocker.

\section{Correlation of polysomnographic parameters with severity of heart failure}

In our study we found significant negative correlation between EF and AHI (central) (Pearson correlation -0.408, $\mathrm{p}$ value $=0.009$ ). This implies that the severity of CSA increases with the decrease in ejection fraction i.e. severity of CSA increases with severity of heart failure. Ejection fraction correlated negatively with both arousal index and desaturation index. Similar results were found in other studies $(6,12)$, which also concluded that central sleep apnea can be considered as a marker of severity of heart failure. (21) When we compared the sleep characteristics of patients of NYHA class 2 and NYHA class 3, we found a significant difference in sleep characteristics of the two groups. Values of AHI (central), O2 desaturation index and arousal index were significantly more in NYHA class 3 as compared to NYHA class 2. Due to frequent arousals, as can be expected, sleep efficiency was lower in NYHA class 3 compared to NYHA class 2 . Similarly minimum O2 saturation achieved was significantly lower in NYHA class 3 compared to NYHA class 2 . The reason for the negative correlation of the ejection fraction and AHI (central) lies in fact that HF causes prolonged circulation time. Prolonged circulatory time, plays a critical role in development of CSR $(13,14)$. The circulatory time in heart failure is prolonged as a result of reduced cardiac output and increased blood volume. This prolonged circulatory time has the potential to create cascades of changes in ventliatory control mechanism that result in development and or maintenance of CSR $(15,16)$. 
Normally, the arterial blood gas changes that occur in pulmonary circulation reach carotid and central chemoceptors promptly, typically within seven seconds.(20) A prolonged circulation time delays this transfer of information by as much as twenty seconds or more. As a result what is usually a negative feedback is transformed into positive feedback system. In this scenario, the arterial blood gas changes that occur during apneic phase may reach the chemoreceptors after apnea phase has terminated, thereby triggering ventilator overshoot during hyperapnea. Arousal associated with CSR may play a role in the pathogenesis of CSR, i.e. the arousal may increase the magnitude of ventilator overshoot during hyperapnea with a subsequent undershoot during hypoventilation.

Our study also showed that patients with heart failure slept significantly less as evidenced by the sleep efficiency. These abnormalities may result in daytime fatigue and sleepiness when measured quantitatively. (17) We found a tendency for greater prevalence of subjective excessive daytime sleepiness and fatigue in patients with sleep disordered breathing. CSA-CSR also resulted in severe arterial oxyhemoglobin desaturation as seen in our study. Periodic breathing also resulted in excessive number of arousals in patients with sleep apnea. Recurrent episodes of nocturnal oxyhemoglobin desaturations and arousals due to periodic breathing, while asleep, could adversely affect the cardiac function by a variety of mechanisms eventually resulting in imbalance between myocardial oxygen delivery and consumption. This study has conclusively found a definite correlation between increasing severity of HF with increasing severity of CSA.

In patients with heart failure, comorbid sleep apnea is most often not tested and consequently subjects are underdiagnosed and not treated.(22) Clearly there is a need for further research, using well designed studies and long term follow up, to fully demonstrate an association of SDB with progression of severity of heart failure. Also large-scale, carefully executed therapeutic studies are needed to determine if the treatment of sleep-related breathing disorders changes the natural history of left ventricular failure. A better understanding of relationship between SDB and heart failure may have important public health implications.

\section{Conclusions}

A fairly high prevalence of sleep-disordered breathing $(57.5 \%)$ was found in patients of heart failure in the present study. With increasing severity of HF a significant worsening of CSA-CSR was observed. The severity of SDB was thus observed to be a surrogate marker of severity of heart failure. The sympathetic activation during multiple arousals and desaturation may be associated with further worsening of HF. The treatment of CSA-CSR may prevent the worsening status of HF. Hence long term randomized and controlled interventions are required to further substantiate these facts.

\section{References}

1. Mann D.L. Heart failure and cor pulmonale, in Harrison's Principles of Internal Medicine, $17^{\text {th }}$ ed. A. S. Fauci et al (eds). New York, McGraw Hill Medical, 2008, pp 1443 1455.

2. Kee K., Sands S.A., Edwards B.A.,Berger P.J., Naughton M.T. positive airway pressure in congestive heart failure. Sleep Med Clin 2010, 5:393-405.

3. Vasan RS, Benjamin EJ, Levy D. Prevalence, clinical features and prognosis of diastolic heart failure: an epidemiologic perspective. J Am Coll Cardiol 1995; 26(7):1565-74.

4. AASM Manual for scoring sleep, 2007.

5. Reddy EV, Kadhiravan T, Mishra HK et al. Prevalence and risk factors of obstructive sleep apnea among middle-aged urban Indians: a community-based study. Sleep Med. 2009 Sep; 10(8):913-8.

6. Javaheri S, Parker TJ, Liming JD, et al. Sleep apnea in 81 ambulatory male patients with stable heart failure. Circulation 1998; 2154-2159.

7. R. Schulz, A. Blau, J. Börgel et al Sleep apnoea in heart failure. Eur Respir J 2007; 29:1201-1205.

8. Sonia Ancoli-Israel, Einat R. Duhamel, Carl Stepnowsky et al. The relationship between congestive heart failure, sleep apnea, and mortality in older men. Chest 2003; 124:1400-1405

9. Köhnlein T, Welte T. Does beta-blocker treatment influence central sleep apnoea? Respir Med 2007; 101:850-853

10. Tamura A, Kawano $Y$, Naono S, Kotoku M, Kadota J. Relationship between beta-blocker treatment and the severity of central sleep apnea in chronic heart failure. Chest $2007 ; 131: 130-135$

11. Sin DD, Fitzgerald F, Parker JD, Newton G, Floras JS, Bradley TD. Risk factors for central and obstructive sleep apnea in 450 men and women with congestive heart failure. Am J Respir Crit Care Med 1999; 160:1101-1106.

12. Tremel F, Pepin JL, Veale D, et al. High prevalence and persistence of sleep apnea in patients referred for acute left ventricular failure and medically treated for 2 months. Eur Heart J. 1999; 20:1201-1209.

13. Tkacova R, Hall MJ, Liu PP, et al, Left ventricular volume in patients with heart failure and Cheyne-Stokes respiration during sleep. Am i respire Crit Care Med 1997; 156:1549. 1555. 
14. Moratara A, Sleight P, Pinna GD, et al. Association between hemodynamic impairment and Cheyne-stokes respiration and periodic breathing in chronic stable congestive heart failure patients secondary to ischemic or idiopathic dilated cardiomyopathy. Am J Cardiol 1999; 84:900-904.

15. Khoo MCK. Theoretical models of periodic breathing in sleep apnea. In: Bradley TD, Floras JS (eds): Sleep Apnea: Implications in cardiovascular and cerebrovascular Disease. New York, Marcel Dekker, 2000:335-384.

16. Guyton AC, Crowell JW: Basic oscillating mechanism of Cheyne-Stokes breathing. Am J of Physiol 1956;187:395398.

17. Hanly O, Zuberi - Khokhar N. Daytime sleepiness in patients with congestive heart failure and Cheyne-Stokes respiration. Chest1995;107:952-958

18. Patidar AB, Andrews GR, Seth S. Prevalence of obstructive sleep apnea, associated risk factors, and quality of life among Indian congestive heart failure patients: A crosssectional survey. J Cardiovasc Nurs. 2011 Mar 2. [Epub ahead of print]

19. Monda C, Scala O, Paolillo S, Savarese G, Cecere M, D'Amore C, Parente A, Musella F, Mosca S, Filardi PP. Sleep apnea and heart failure: pathophysiology, diagnosis and therapy. G Ital Cardiol (Rome). 2010 Nov; 11(11):81522 .

20. Kasai T, Bradley TD. Obstructive sleep apnea and heart failure: pathophysiologic and therapeutic implications. J Am Coll Cardiol. 2011 Jan 11; 57(2):119-27

21. Varol E, Akcay S, Ozaydin M, Ozturk O, Cerci SS, Sahin U. Influence of obstructive sleep apnea on left ventricular mass and global function: sleep apnea and myocardial performance index. Heart Vessels 2010 Sep; 25(5):400-4.

22. Javaheri S, Caref EB, Chen E, Tong KB, Abraham WT. Sleep apnea testing and outcomes in a large cohort of medicare beneficiaries with newly diagnosed heart failure. Am J Respir Crit Care Med. 2011 Feb 15;183(4):539-46.

23. Sleep Related Breathing Disorders In: Winkelman J, Kotagal $\mathrm{S}$, Olson $\mathrm{E}$ et al.(eds.) The International Classification of Sleep Disorders Diagnostic\& Coding Manual. American Academy of Sleep Medicine, Illinois, 2006:47-56.

24. Javaheri S. Central sleep apnea. Clin Chest Med 2010; $31: 235-248$

Indian Journal of Sleep Medicine (IJSM), Vol. 5, No. 4, 2010 